\title{
Possible use of ail and foxA polymorphisms for detecting pathogenic Yersinia enterocolitica
}

\author{
Ying Huang ${ }^{1+}$, Xin Wang ${ }^{1 \dagger}$, Zhigang Cui ${ }^{1+}$, Yuhuan Yang ${ }^{2}$, Yuchun Xiao ${ }^{1}$, Liuying Tang ${ }^{3}$, Biao Kan ${ }^{1}$, Jianguo Xu', \\ Huaiqi Jing ${ }^{1 *}$
}

\begin{abstract}
Background: Yersinia enterocolitica is an enteric pathogen that invades the intestinal mucosa and proliferates within the lymphoid follicles (Peyer's patches). The attachment invasion locus (ail) mediates invasion by $Y$. enterocolitica and confers an invasive phenotype upon non-invasive E. coli; ail is the primary virulence factor of Y. enterocolitica. The ferrioxamine receptor (foxA) located on the $Y$. enterocolitica chromosome, together with its transport protein, transports a siderophore specific for ferric ion. Currently, ail is the primary target gene for nucleic acid detection of pathogenic $Y$. enterocolitica.

Results: The genes ail and foxA in 271 pathogenic and 27 non-pathogenic $Y$. enterocolitica strains isolated from China and 10 reference strains were sequenced, aligned, compared to the ail and foxA sequences of Yersinia enterocolitica subsp. enterocolitica 8081 (Genbank: NC_008800), and analyzed for sequence polymorphism. The ail from the 282 strains showed 3 sequence patterns: 277 strains of serotypes O:3, O:9 and O:5, 27 with identical nucleic acid sequences formed pattern A1; 4 strains of serotype 1B/O:8 with identical nucleic acid sequences formed pattern $A 2$; and one Chinese isolate 2/O:9 formed pattern A3. In the primary coding region of the foXA ORF (Genebank: X60447 nt 433-1866; nt 28 to 1,461 in the ORF), the sequences formed 3 groups and were further divided into 8 sequence patterns.

Conclusion: The ail and foxA loci of pathogenic Y. enterocolitica have been analyzed. The ail sequence was highly conserved among the same serotype strains from different sources; and foxA was highly conserved among the pathogenic strains, although there was some sequence diversity. Fewer strains were used from outside China, which is a limitation of the study.
\end{abstract}

\section{Background}

Yersinia enterocolitica is a food-borne pathogen [1] that causes a broad spectrum of clinical syndromes. A primary characteristic of the microorganism is that it penetrates the intestinal epithelial cells and replicates in lymphatic nodules, causing a wide variety of clinical and immunological manifestations [2-4]. All pathogenic $Y$. enterocolitica strains harbor ail, which is different from the inv sequence (which encodes a protein of similar function), and renders $Y$. enterocolitica capable of invading the intestinal epithelium. In addition, the Ail protein confers a serum resistance phenotype on $Y$. enterocolitica [5]. In contrast to inv,

\footnotetext{
* Correspondence: jinghuaiqi@icdc.cn

† Contributed equally

'National Institute for Communicable Disease Control and Prevention, Chinese Center for Disease Control and Prevention, State Key Laboratory for Infectious Disease Prevention and Control, 102206, Beijing, China Full list of author information is available at the end of the article
}

which exists in non-pathogenic as well as pathogenic strains of $Y$. enterocolitica, ail only exists in $Y$. enterocolitica strains epidemiologically related to human disease [6], and is therefore an important virulence marker. Environmental isolates not associated with disease have a nonfunctional inv and no ail [7].

Ferric ion uptake is essential for bacterial growth and survival. The supply of iron and production of the siderophore transport system is a central factor in infections with Yesinia pestis and Y. enterocolitica. Pathogenic $Y$. enterocolitica can be divided into 2 groups, those producing the siderophore, such as biotype $1 \mathrm{~B} / \mathrm{O}: 8$, and those producing no siderophore, as in serotypes $\mathrm{O}: 3$ and $\mathrm{O}: 9$ [8]; the latter take up ferric ion using ectogenic siderophores, such as ferrioxamin B and ferrioxamin E [9]. The 2 groups have different ferric ion uptake abilities, which may explain the differences in virulence among serotypes [10]. A $77 \mathrm{kDa}$ receptor on the $Y$. enterocolitica outer membrane [11]
Ciomed Central 
combines with ferrioxamin to take up ferric ion rapidly [12]. This process is energy-dependent and requires the action of the TonB protein, part of a complex known as the Ton system. This complex undergoes a conformational change driven by the proton motive force (PMF), which interacts with the outer membrane receptors and activates transport [13]. The FoxA receptor of $Y$. enterocolitica, the ferrochrome receptor and the TonB-dependent receptor share high amino acid homology $[14,15]$. The fox $A$ was chosen for study because it exists in all $Y$. enterocolitica strains.

Using polymorphic gene analysis, we show that combined detection of ail and foxA confirms the identity of pathogenic $Y$. enterocolitica.

\section{Methods}

\section{Bacterial strains and identification of biotype and} serotype

We chose 271 pathogenic and 27 non-pathogenic $Y$. enterocolitica strains isolated from diarrhea patients, animals, food and the environment in China. They included 205 strains of serotype O:9, 72 of serotype O:3, 10 of serotype O:8, 5 of serotype O:5, 3 of serotype O:6, 30 and 3 of undetermined serotype (Table 1), together with 11 reference strains from Europe, the United States and Japan (Table 2). The serotypes, biotypes and pathogenesis of these strains were determined as previously described [16-18].

\section{Primer nucleotide sequences}

The primers for ail and foxA were designed in our laboratory, referencing sequences from GenBank (ail: M29945, foxA: X60447), and synthesized by Shanghai Sangon Biological Engineering \& Technology and Service Co., Ltd, China. The primers for ail amplify the entire ORF, while those for fox $A$ amplify the ORF coding region from nt 28 to nt 1,461 (Table 3).

\section{PCR, DNA sequencing and sequence analysis}

Bacteria were cultured as previously described [18]. The bacterial DNA was extracted using a Blood \& Tissue Kit (QIAGEN, USA). PCR was performed in a 200 $\mu \mathrm{l}$ volume containing $10 \mathrm{ng}$ DNA template, 5U Taq DNA polymerase (TaKaRa, China), $0.2 \mathrm{mM}$ of each dNTP, $1 \mu \mathrm{M}$ of each forward and reverse primer, 1.5 $\mathrm{mM} \mathrm{MgCl}_{2}, 50 \mathrm{mM} \mathrm{KCl}$, and $10 \mathrm{mM}$ Tris- $\mathrm{HCl}(\mathrm{pH}$ 8.3). Thermal cycling was done in a MJ PTC200 (BioRad, USA) and the conditions were: one cycle of denaturation at $94^{\circ} \mathrm{C}$ for $5 \mathrm{~min}$, followed by 25 cycles of melting at $94^{\circ} \mathrm{C}$ for $15 \mathrm{~s}$, annealing for $30 \mathrm{~s}$ at various temperatures depending on the primers used (Table 3), elongation at $72^{\circ} \mathrm{C}$ for $30 \mathrm{~s}$, and a final extension at $72^{\circ} \mathrm{C}$ for $10 \mathrm{~min}$. Five microliters of PCR product was electrophoresed on a $1.5 \%$ agarose gel. The gel image was captured using a Gel Documentation 2000 (BioRad, USA).

The specific PCR products were purified using a Gel Extraction Kit (QIAGEN, USA) and sequenced using an ABI PRISM ${ }^{\bullet}$ BigDyeTM Terminator cycle sequencing Ready Reaction Kit with AmpliTag DNA Polymerase, following the manufacturer's instructions, and an ABI PRISM $^{\oplus}$ 377XL DNA Sequencer (Applied Biosystems, Foster City, CA, USA) at TaKaRa Biotechnology (Dalian) Co., Ltd. The sequences were aligned with the reference sequences. Nucleotide sequence alignments and cluster tree construction were performed using Clustal X (Version 1.8) and MEGA (Version 4).

\section{Results}

General features of ail and foxA

ail is located on the $Y$. enterocolitica chromosome where the ORF encodes a peptide of 178 amino acids, MW: 19,548 Da [19]. There is a typical prokaryotic signal sequence at the $\mathrm{N}$-terminus of the peptide [20] with

Table 1 Bio-serotypes of the $298 \mathrm{Y}$. enterocolitica isolates from China

\begin{tabular}{|c|c|c|c|c|c|c|c|c|c|c|c|c|c|}
\hline \multirow[t]{2}{*}{ Host } & \multicolumn{6}{|c|}{ Pathogenic Strains } & \multicolumn{7}{|c|}{ Non-pathogenic Strains } \\
\hline & 2/0:9 & 3/0:9 & 2/0:3 & 3/0:3 & 4/0:3 & Total & 1A/0:3 & 1A/0:9 & 1A/0:8 & 1A/0:5 & $1 \mathrm{~A} / 0: 6,30$ & 1A/Un* & Total \\
\hline Patient & 6 & 2 & 1 & 4 & 1 & 14 & & & & 5 & 3 & & 8 \\
\hline Swine & 83 & 3 & & 46 & & 132 & 1 & 2 & 4 & & & & 7 \\
\hline Dog & 3 & & & 13 & & 16 & & & & & & 1 & 1 \\
\hline Fowl & 1 & 1 & & 1 & & 3 & 1 & 1 & & & & & 2 \\
\hline Goat & & & & 2 & & 2 & & & 2 & & & & 2 \\
\hline Cattle & & 1 & & & & 1 & & 1 & 2 & & & & 3 \\
\hline Fish & & & & 1 & & 1 & & & & & & & 0 \\
\hline Rat & 96 & 1 & & 1 & & 98 & & & & & & 1 & 1 \\
\hline Rabbit & 1 & & & & & 1 & & & & & & & 0 \\
\hline Food & 1 & 1 & & & & 2 & & & 2 & & & 1 & 3 \\
\hline Environment & 1 & & & & & 1 & & & & & & & 0 \\
\hline Total & 192 & 9 & 1 & 68 & 1 & 271 & 2 & 4 & 10 & 5 & 3 & 3 & 27 \\
\hline
\end{tabular}

*: undetermined serotype 
Table 2 Sources of the 11 reference strains

\begin{tabular}{|c|c|c|c|}
\hline $\begin{array}{l}\text { Strains } \\
\text { No. }\end{array}$ & Bioserotype & Location & Source \\
\hline 52203 & 4/0:3 & $\begin{array}{l}\text { The Pasteur Institute, } \\
\text { France }\end{array}$ & Purchased from the Pasteur Institute by the Institute of Chinese Biomedicine. \\
\hline 52212 & 4/0:9 & & \\
\hline 52211 & 1B/O:8 & & \\
\hline Pa40134 & 4/O:3 & Japan & $\begin{array}{l}\text { Provided by Dr. H. Fukushima (Public Health Institute of Shimane Prefecture, Matsue, } \\
\text { Japan). }\end{array}$ \\
\hline ye3vp-/03 & 3/0:3 & & \\
\hline ye3vp5/03 & 3/0:3 & & \\
\hline ye4/03 & 4/O:3 & & \\
\hline D92 & 2/0:5,27 & & \\
\hline Pa12986 & 1B/O:8 & & \\
\hline Ye92010 & $1 \mathrm{BO}: 8$ & & \\
\hline 8081 & 1B/O:8 & \multicolumn{2}{|c|}{$\begin{array}{l}\text { Complete genome sequence of the highly pathogenic Yersinia enterocolitica subsp. enterocolitica } 8081 \text { (Genbank: } \\
\text { NC_008800). }\end{array}$} \\
\hline
\end{tabular}

a cleavage site between residues 23 and 24, where the first 23 amino acids act as a signal sequence [19].

foxA has an ORF of 2,129 bp encoding a protein of 710 amino acids, MW: 78,565 Da. The first 26 amino acids are a signal sequence, and a mature protein of 684 aa, MW: $75,768 \mathrm{Da}$, is formed after cleavage [14]. There is a sequence ahead of fox $A$ with homology to the putative ferric ion uptake regulator (Fur) of Yersinia [21]. The expression of foxA may be regulated by iron via the Fur protein, as in other known siderophore receptors [14]. Fur may be a transcription inhibition protein acting on the ferric regulation promoter using $\mathrm{Fe}^{2+}$-dependent DNA binding activity homologous to that in E. coli [22-25].

\section{Analysis of ail}

The entire ail ORF for 271 pathogenic Y. enterocolitica strains isolated from China and 10 reference strains were analyzed and compared to strain 8081 . The data showed that all the strains can be divided into 3 sequence patterns. The Chinese isolates, 270 strains (70 of serotype O:3 and 200 of serotype O:9) and 7 reference strains (5 of O:3, one of O:9 and one of O:5,27), were sequentially identical and formed pattern A1. Four highly pathogenic strains of serotype $1 \mathrm{~B} / \mathrm{O}: 8$ showed identical sequences and formed pattern A2. Compared to pattern A1, pattern A2 showed 21 base mutations among which 9 were sense and 12 were nonsense mutations. In addition, one pathogenic Chinese isolate O:9 serotype (isolated from the tongue of a rat in Ningxia, 1997) showed 3 base mutations compared to the entire ail of pattern A1, one sense and 2 nonsense; it formed pattern A3 (Fig. 1). This new ail genotype was submitted to Genbank and given the accession number GU722202.

\section{Analysis of foxA}

Analysis of the primary coding region of fox $A$ from nt 28 to nt 1,461 in 271 pathogenic $Y$. enterocolitica strains isolated from China and 11 reference strains showed that all the strains can be divided into 3 groups including 8 sequence patterns (Fig. 2). Group I comprised patterns F1, F2 and F3 and included 201 serotype O:9 strains isolated from China and 2 reference strains (one strain O:9 and one O:5,27). Among these, 199 strains of O:9 and 2 reference strains showing identical sequences formed pattern F1, and 2 other strains of O:9 with 2 and 3 base mutations formed patterns F2 and F3. Group II comprised patterns F4 and F5, and included 70 Chinese isolates and 5 reference strains of serotype O:3. Sixty-nine serotype O:3 strains (67 Chinese isolates and2 reference strains) showing identical sequences formed

Table 3 Primer sequences and annealing temperatures for ail and foxA

\begin{tabular}{|c|c|c|c|c|c|c|}
\hline \multicolumn{2}{|c|}{ Target gene and primer direction } & \multirow{2}{*}{$\frac{\text { Primer Sequences }\left(5^{\prime} \rightarrow 3^{\prime}\right)}{\text { GGT TAT TGT ATT AGT ATT GTT }}$} & \multirow{2}{*}{$\begin{array}{l}\text { GenBank no. } \\
\text { M29945 }\end{array}$} & \multirow{2}{*}{$\frac{\text { Location (nt) }}{446-466}$} & \multirow{2}{*}{$\begin{array}{l}\text { Amplicon length } \\
585 \mathrm{bp}\end{array}$} & \multirow{2}{*}{$\begin{array}{l}\text { Annealing temp. } \\
57^{\circ} \mathrm{C}\end{array}$} \\
\hline ail & Forward & & & & & \\
\hline & Reverse & CAG GTG GGT TIT CAC TAT CTG & & $1031-1051$ & & \\
\hline \multirow[t]{2}{*}{ foxA } & Forward & CTC TGC GGA AGA TAA CTA TG & $\times 60447$ & $389-408$ & $1532 \mathrm{bp}$ & $58^{\circ} \mathrm{C}$ \\
\hline & Reverse & ATC CGG GAA TAA ACT TGG CGT A & & 1899-1920 & & \\
\hline
\end{tabular}




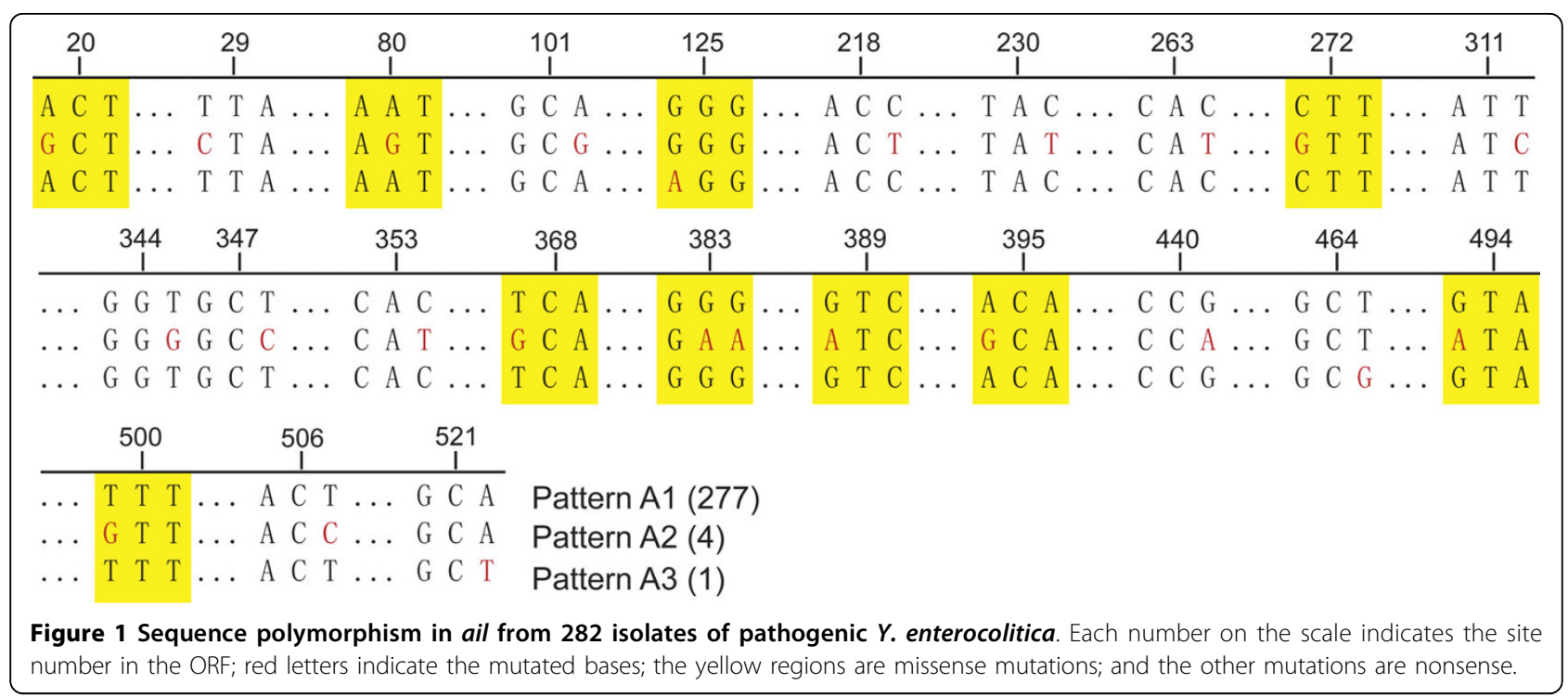

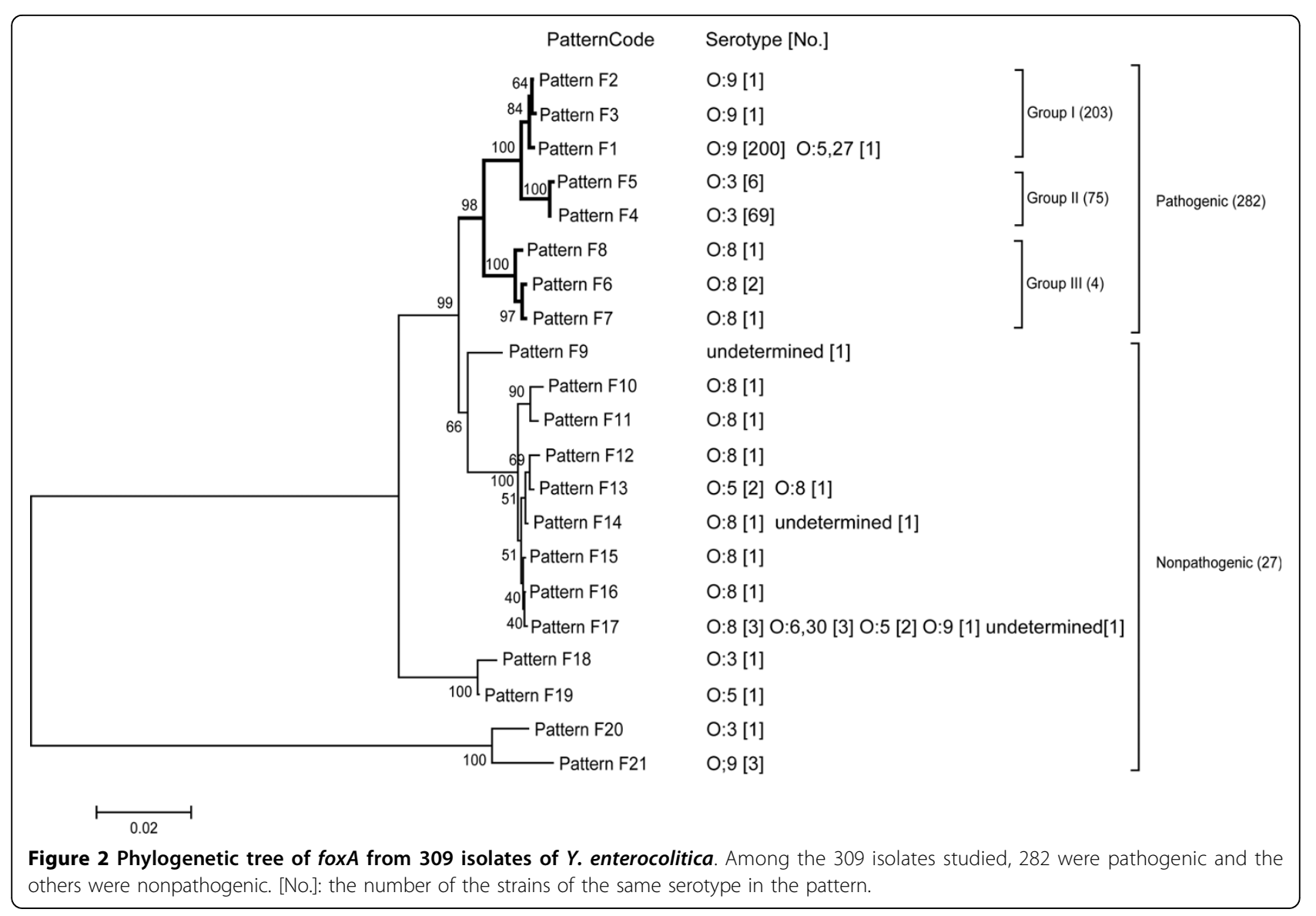




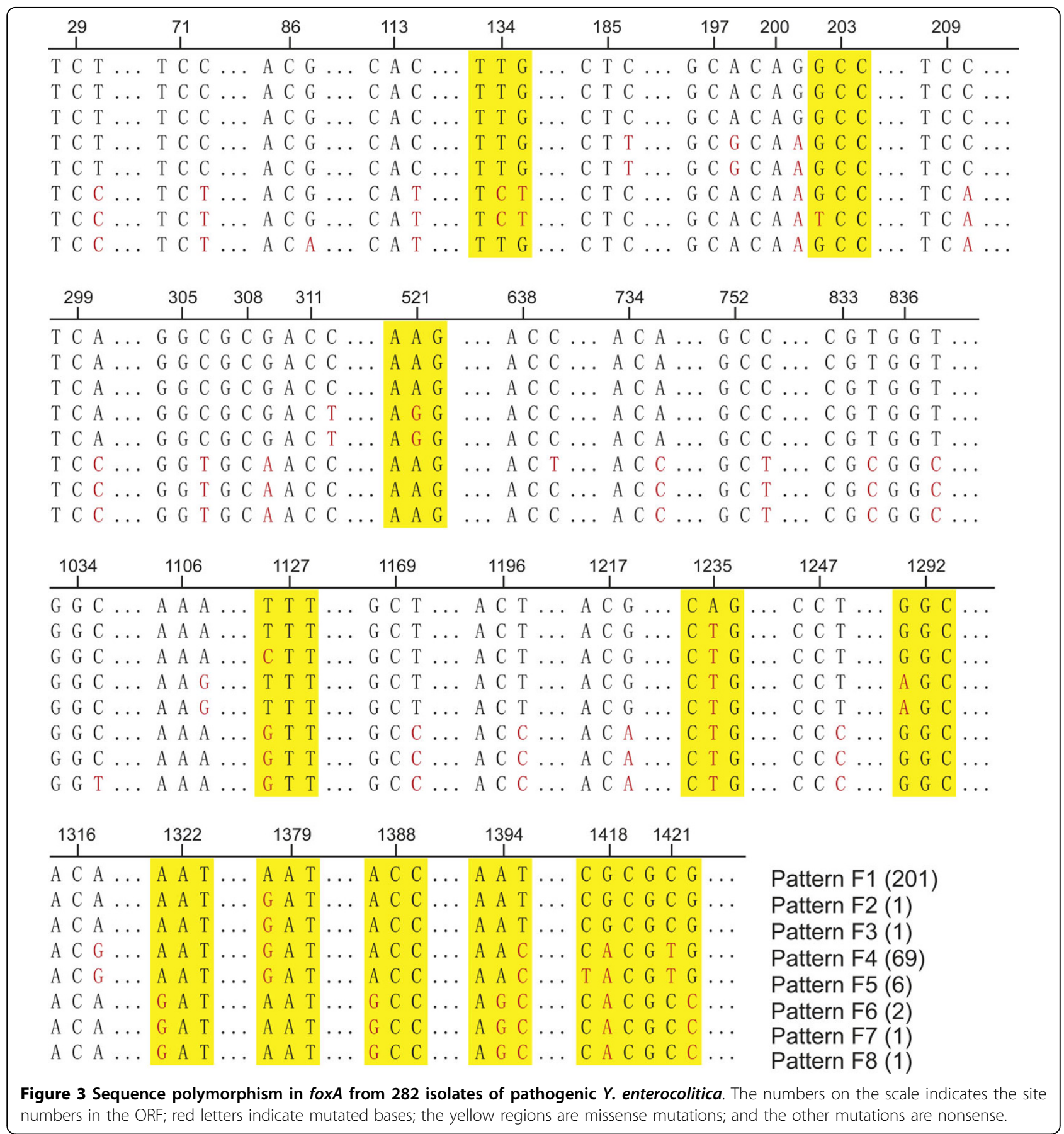

pattern F4; and 6 other strains of O:3 had one base mutation and formed pattern F5. Group III comprised five reference strains including patterns F6, F7 and F8. Pattern F6 (2 Japanese strains) had 2 base mutations compared to pattern F7 (52211). Compared to pattern F7, pattern F8 (8081) had 5 base mutations (Fig. 3).

To analyze foxA polymorphism in $Y$. enterocolitica overall, we chose 27 strains of non-pathogenic $Y$. enterocolitica as controls (Table 1 ). The results showed 13 sequence patterns for the 27 strains with 10's to 100's more polymorphic sites and no apparent regularity. This indicated that fox $A$ was less polymorphic and more conserved in pathogenic strains than in non-pathogenic strains.

\section{Discussion}

Only pathogenic $Y$. enterocolitica contains ail, which confers a bacterial invasion and serum resistance 
phenotype, that is an important virulence marker on the chromosome [6,19]. The entire ORF of ail was sequenced and analyzed from strains from different sources and biotypes and serotypes. The data showed that the 282 pathogenic $Y$. enterocolitica formed 3 sequence patterns (Fig. 1); the strains were pathogenic $\mathrm{O}: 3$ and O:9 isolated from various hosts in China and the reference strains. Only one Chinese isolate formed pattern A3, a new ail genotype submitted to Genbank and given the GenBank accession number GU722202. When it was compared to the sequence of pattern A1, three base mutations were found, one sense and two nonsense. We presume that pathogenic $Y$. enterocolitica had 2 original ail patterns, A1 represented in serotypes $\mathrm{O}: 3$ and $\mathrm{O}: 9$ and $\mathrm{A} 2$ represented in bio-serotype 1B/O:8; pattern A3 may be a mutation of A1. Pathogenic $Y$. enterocolitica can be divided into a high-pathogenicity group (Y. enterocolitica biogroup 1B) and a low-pathogenicity group ( $Y$. enterocolitica biogroups 2 to 5 ) on the basis of the lethal infectious dose in the mouse model [26]. The typing of ail in this study is consistent with this grouping of pathogenic strains. Therefore, we presumed that lethality in mice (50\% lethal dose $<1,000$ microorganisms) not only depends on the presence of the yersiniabactin $(y b t)$ locus, which carries the genes for biosynthesis, transport and regulation of the siderophore yersiniabactin [8,27-29], but is also closely related to the ail sequence. We conjecture that synergism with ail is necessary for $Y$. enterocolitica pathogenesis.

ail is not only an important virulence gene for pathogenic $Y$. enterocolitica, but also harbors highly conserved sequences, mutation of which may change the virulence of the bacterium. For instance, in the 1B/O:8 strain, which is highly lethal to mice, the ail belongs to pattern A2, while ail in other pathogenic bioserotype strains belongs to pattern A1. So we believe that a change in ail is closely related to the pathogenesis of the strain. A pathogenic O:9 strain isolated from Cricetulus triton in Ningxia contains ail pattern A3, the sequence of which has 3 site mutations, only one being a sense mutation. Further study is needed to see whether amino acid change alters the function of Ail protein or bacterial virulence.

Analysis of the 1,434 base pairs of the fox $A$ primary coding region showed that the fox $A$ sequence correlated with the biotype and serotype of pathogenic $Y$. enterocolitica. Comparing the primary sequences of groups I and II, 13 base mutations at fixed positions were found; 5 were sense and 8 were nonsense mutations, indicating that the primary difference in the pathogenic $Y$. enterocolitica foxA was located in these 13 sites. Strain 8081 showed 26 base mutations compared to F1 and 31 compared to F4. From these findings we presume that pathogenic $0: 3$ and $0: 9$ have similar fox $A$ sequences
(Fig. 2) and mutation sites additional to strain 8081 bioserotype $1 \mathrm{~B} / \mathrm{O}: 8$ (Fig. 3). Thus, there is a correlation between pathogenesis and the different bio-serotypes of $Y$. enterocolitica. More mutation sites and no obvious regulation were found in non-pathogenic $Y$. enterocolitica fox $A$, although some strains showed an identical fox $A$ sequence type (Fig. 2). The identical sequence patterns of the pathogenic $Y$. enterocolitica strains isolated from different areas, at different times and from different host sources show the fox $A$ sequence pattern to be closely correlated to pathogenesis. Unfortunately, fewer strains from outside China were used, which is a limitation of the study and needs adding strains for future study.

ail is a primary marker for pathogenic $Y$. enterocolitica and is an important tool for detecting it, making it a very important gene to analyze. Some scholars have established a real-time PCR assay to detect $Y$. enterocolitica using ail or ystA as the target gene [30-33]. According to the current identification standards, strains having no ail and harboring $y s t B$ isolated from diarrhea patients are classified as non-pathogenic. However, other researchers believe that strains harboring $y s t B$ are pathogenic and cause the diarrhea, as inferred from epidemiology and the etiology of disease outbreaks [34,35]. Therefore, it is necessary to detect other conserved genes as a complement to these markers because reliance on only ail and $y s t A$ to detect pathogenesis remains questionable. We chose to detect fox $A$, which is found in both pathogenic and non-pathogenic $Y$. enterocolitica. The results showed that both ail and foxA were conserved together in pathogenic strains and can therefore be used to confirm the detection of pathogenic $Y$. enterocolitica. Currently, we are attempting to extract bacterial DNA from clinical specimens to detect foxA in order to identify $Y$. enterocolitica directly from humans and other animals; and we have some preliminary data (unpublished).

Almost all $Y$. enterocolitica carry foxA while pathogenic strains carry ail. It is very important for real-time PCR detection of $Y$. enterocolitica to study sequence polymorphism in ail and foxA. It will be helpful to design specific primers and probes in the conserved region in order to develop real-time or traditional PCR methods. We are trying to establish a duplex real-time PCR to detect $Y$. enterocolitica from clinical samples and to confirm its pathogenicity. Designing specific primers for fox $A$ and ail in a combined detection system is valuable for increasing sensitivity and specificity in the detection of pathogenic $Y$. enterocolitica.

\section{Conclusion}

Analysis of polymorphisms in ail and foxA of pathogenic $Y$. enterocolitica strains from different times and regions 
showed ail to be an important virulence gene for pathogenic $Y$. enterocolitica, and that it has a highly conserved sequence. The gene encoding the ferrioxamine receptor, fox $A$, is also conserved in pathogenic strains, where 2 primary sequence patterns were found. More strains from outside China are needed for further study.

\section{Acknowledgements}

This work was supported by National Natural Science Foundation of China (General Project, No. 30970094).and National Sci-Tech key project (2009ZX10004-201, 2009ZX10004-203).

We thank Dr. Jim Nelson for critical reading of our manuscript.

\section{Author details}

'National Institute for Communicable Disease Control and Prevention, Chinese Center for Disease Control and Prevention, State Key Laboratory for Infectious Disease Prevention and Control, 102206, Beijing, China. ${ }^{2}$ Fujian Provincial Centre for Disease Control and Prevention, 350001, Fuzhou, China. ${ }^{3}$ National Institute for Viral Disease Control and Prevention, Chinese Center for Disease Control and Prevention, 100052, Beijing, China.

\section{Authors' contributions}

YH did most of the PCR work and DNA sequencing. XW analyzed the sequences. ZC did the data clustering and construction of phylogenetic trees. $Y Y$ and $Y X$ identified the biotypes and serotypes of strains. LT wrote the paper. BK and XJ participated in discussion of the study. $\mathrm{HJ}$ designed and coordinated the study and drafted the manuscript. All the authors read and approved the final manuscript.

Received: 4 March 2010 Accepted: 7 August 2010

Published: 7 August 2010

\section{References}

1. Bottone EJ: Yersinia enterocolitica: a panoramic view of a charismatic microorganism. CRC Crit Rev Microbiol 1977, 5:211-241

2. Pepe JC, Miller VL: Yersinia enterocolitica invasin: a primary role in the initiation of infection. Proc Natl Acad Sci USA 1993, 90:6473-6477.

3. Cover TL, Aber RC: Yersinia enterocolitica. N Engl J Med 1989, 321:16-24.

4. Grutzkau A, Hanski $C$, Hahn H, Riecken EO: Involvement of $M$ cells in the bacterial invasion of Peyer's patches: a common mechanism shared by Yersinia enterocolitica and other enteroinvasive bacteria. Gut 1990 31:1011-1015.

5. Pierson DE, Falkow S: The ail gene of Yersinia enterocolitica has a role in the ability of the organism to survive serum killing. Infect Immun 1993, 61:1846-1852.

6. Miller VL, Farmer JJ III, Hill WE, Falkow S: The ail locus is found uniquely in Yersinia enterocolitica serotypes commonly associated with disease. Infect Immun 1989, 57:121-131.

7. Pierson DE, Falkow S: Nonpathogenic isolates of Yersinia enterocolitica do not contain functional inv-homologous sequences. Infect Immun 1990 58:1059-1064

8. Heesemann J: Chromosomal-encoded siderophores aer required for mouse virulence of enteropathogenic Yersinia species. FEMS Microbiol Letts 1987, 48.229-233.

9. Baumler A, Koebnik R, Stojiljkovic I, Heesemann J, Braun V, Hantke K: Survey on newly characterized iron uptake systems of Yersinia enterocolitica. Zentralbl Bakteriol 1993, 278:416-424

10. Bakour R, Balligand G, Laroche Y, Cornelis G, Wauters G: A simple adultmouse test for tissue invasiveness in Yersinia enterocolitica strains of low experimental virulence. J Med Microbiol 1985, 19:237-246.

11. Baumler AJ, Hantke K: A lipoprotein of Yersinia enterocolitica facilitates ferrioxamine uptake in Escherichia coli. J Bacteriol 1992, 174:1029-1035

12. Perry RD, Brubaker RR: Accumulation of iron by yersiniae. J Bacteriol 1979, 137:1290-1298.

13. Faraldo-Gomez JD, Sansom MS: Acquisition of siderophores in gramnegative bacteria. Nat Rev Mol Cell Biol 2003, 4:105-116.
14. Baumler AJ, Hantke K: Ferrioxamine uptake in Yersinia enterocolitica: characterization of the receptor protein FoxA. Mol Microbiol 1992, 6:1309-1321.

15. Kornreich-Leshem H, Ziv C, Gumienna-Kontecka E, rad-Yellin R, Chen $Y$, Elhabiri M, brecht-Gary AM, Hadar $Y$, Shanzer A: Ferrioxamine B analogues: targeting the FoxA uptake system in the pathogenic Yersinia enterocolitica. J Am Chem Soc 2005, 127:1137-1145.

16. Bottone EJ: Yersinia enterocolitica: the charisma continues. Clin Microbiol Rev 1997, 10:257-276.

17. Thoerner P, Bin Kingombe Cl, Bogli-Stuber K, Bissig-Choisat B, Wassenaar TM, Frey J, Jemmi T: PCR detection of virulence genes in Yersinia enterocolitica and Yersinia pseudotuberculosis and investigation of virulence gene distribution. Appl Environ Microbiol 2003, 69:1810-1816.

18. Wang X, Qiu H, Jin D, Cui Z, Kan B, Xiao Y, Xu Y, Xia S, Wang H, Yang J, et al: O:8 serotype Yersinia enterocolitica strains in China. Int J Food Microbiol 2008, 125:259-266.

19. Miller VL, Bliska JB, Falkow S: Nucleotide sequence of the Yersinia enterocolitica ail gene and characterization of the Ail protein product. $J$ Bacteriol 1990, 172:1062-1069.

20. Michaelis S, Beckwith J: Mechanism of incorporation of cell envelope proteins in Escherichia coli. Annu Rev Microbiol 1982, 36:435-465.

21. Staggs TM, Perry RD: Identification and cloning of a fur regulatory gene in Yersinia pestis. J Bacteriol 1991, 173:417-425.

22. Bagg A, Neilands JB: Ferric uptake regulation protein acts as a repressor, employing iron (II) as a cofactor to bind the operator of an iron transport operon in Escherichia coli. Biochemistry 1987, 26:5471-5477.

23. de $L, V$, Wee $S$, Herrero M, Neilands JB: Operator sequences of the aerobactin operon of plasmid ColV-K30 binding the ferric uptake regulation (fur) repressor. J Bacteriol 1987, 169:2624-2630.

24. Escolar L, Perez-Martin J, de L, V: Coordinated repression in vitro of the divergent fepA-fes promoters of Escherichia coli by the iron uptake regulation (Fur) protein. J Bacteriol 1998, 180:2579-2582.

25. Escolar $L$, de $L, V$, Perez-Martin J: Metalloregulation in vitro of the aerobactin promoter of Escherichia coli by the Fur (ferric uptake regulation) protein. Mol Microbiol 1997, 26:799-808.

26. Carter PB: Pathogenecity of Yersinia enterocolitica for mice. Infect Immun $1975,11: 164-170$

27. Heesemann J, Algermissen B, Laufs R: Genetically manipulated virulence of Yersinia enterocolitica. Infect Immun 1984, 46:105-110.

28. Heesemann J, Hantke K, Vocke T, Saken E, Rakin A, Stojiljkovic I, Berner R: Virulence of Yersinia enterocolitica is closely associated with siderophore production, expression of an iron-repressible outer membrane polypeptide of $65,000 \mathrm{Da}$ and pesticin sensitivity. Mol Microbiol 1993, 8:397-408

29. Pelludat C, Rakin A, Jacobi CA, Schubert S, Heesemann J: The yersiniabactin biosynthetic gene cluster of Yersinia enterocolitica: organization and siderophore-dependent regulation. J Bacteriol 1998, 180:538-546

30. Boyapalle S, Wesley IV, Hurd HS, Reddy PG: Comparison of culture, multiplex, and $5^{\prime}$ nuclease polymerase chain reaction assays for the rapid detection of Yersinia enterocolitica in swine and pork products. J Food Prot 2001, 64:1352-1361

31. Jourdan AD, Johnson SC, Wesley IV: Development of a fluorogenic 5 nuclease PCR assay for detection of the ail gene of pathogenic Yersinia enterocolitica. Appl Environ Microbiol 2000, 66:3750-3755.

32. Lambertz ST, Nilsson C, Hallanvuo S, Lindblad M: Real-time PCR method for detection of pathogenic Yersinia enterocolitica in food. Appl Environ Microbiol 2008, 74:6060-6067.

33. Vishnubhatla A, Fung DY, Oberst RD, Hays MP, Nagaraja TG, Flood SJ: Rapid $5^{\prime}$ nuclease (TaqMan) assay for detection of virulent strains of Yersinia enterocolitica. Appl Environ Microbiol 2000, 66:4131-4135.

34. Singh I, Virdi JS: Production of Yersinia stable toxin (YST) and distribution of yst genes in biotype 1A strains of Yersinia enterocolitica. J Med Microbiol 2004, 53:1065-1068.

35. Singh I, Virdi JS: Interaction of Yersinia enterocolitica biotype 1 A strains of diverse origin with cultured cells in vitro. Jpn J Infect Dis 2005, 58:31-33.

doi:10.1186/1471-2180-10-211

Cite this article as: Huang et al:: Possible use of ail and foxA polymorphisms for detecting pathogenic Yersinia enterocolitica. BMC Microbiology 2010 10:211 